Summary

In this brief review a manual micro-Kjeldahl digestion procedure is outlined, which is satisfactory for most substances of biological origin. The final step of ammonia determination may be achieved by steam distillation and titration, if only one or two samples are to be processed. If up to say thirty or forty analyses/d are to be analysed then the semi-automated procedure has considerable advantages. If more than 50 and up to roo samples must be analysed/d then the suitability of the fully automated method should be carefully considered. Depending on availability of staff and digestion equipment such large numbers could also be processed by the semi-automated procedure outlined above.

\title{
REFERENCFS
}

Alexander, R. H. (1969). Lab. Pract. 18,63.

Bradstreet, R. B. (1957). Analyt. Chem. 29, 944.

Bradstreet, R. B. (1965). The Kjeldahl Method for Organic Nitrogen. New York and London: Academic Press.

Dumas, J. B. (1831). Annls Chim. Phys. 2, 198.

Fleck, A. (r967). Proc. Ass. clin. Biochem. 4, 212.

Fleck, A. \& Munro, H. N. (1965). Clinica chim. Acta 11, 2.

Gehrke, C. W., Kaiser, F. E. \& Ussary, J. P. (1968). F. Ass. Off. Analyt. Chem. 51, zoo.

Hiller, A., Plazin, J. \& Van Slyke, D. D. (1948). F. biol. Chem. 176, 1401.

Jacobs, S. (1965). Meth. biochem. Analysis 13, 241.

Jacobs, S. C. (1968). F. clin. Path. 21, 218.

Kjeldahl, J. (1883). Z. analyt. Chem. 22, 366.

Lake, G. R., McCutchan, P., Van Meter, R. \& Neel, J. C. (1951). Analyt. Chem. 23, 1634.

McKenzie, H. A. \& Wallace, H. S. (1954). Aust. F. Chem. 7, 55.

Mann, L. T. Jr (1963). Analyt. Chem. 35, 2179.

Marten, J. F. \& Catanzaro, E. W. (1966). Analyst, Lond. 9r, 42.

Munro, Ir. N. \& Fleck, A. (1969). In Manmalian Protein Metabolism Vol. 3 , Ch. 30 [H. N. Munro, editor]. New York: Academic Press.

Patel, S. M. \& Sreenivasan, A. (1948). Analyt. Chem. 20, 63.

Peters, J. P. \& Van Slyke, D. D. (1932). Quantitative Clinical Chemistry Vol. 2. Methods. London: Ballière, Tindall \& Cox.

Searcy, R. L., Gough, G. S., Korotzcr, J. I. \& Bergquist, L. M. (I96r). Am. J. med. Technol. $27,255$. Skendzel, L. P. \& Muelling, R. J. (1967). New Engl. F. Med. 277, 180.

\section{Influence of transcription techniques on the precision of autoanalyser assays}

By W. J. Tilstone and A. Fleck, Department of Biochemistry, Royal Infirmary, Glasgow, $\mathrm{C}_{4}$

Most workers whose interests include methodology are aware that errors may be introduced at any stage involving operator intervention. With the development of the autoanalyser, operator errors are considerably reduced but where transcription is manual errors will always occur. The magnitude of the error in any given sample may be small and is probably difficult to measure, but there can be individual samples where the error will be appreciable. It is the intention of this communication to quantify the errors involved in various transcription techniques: the work was 
done on results from a slightly modified version of the autoanalyser nitrogen method described by Fleck (1967).

Almost all of the results presented deal with precision, that is the reproducibility of the assay. It is very difficult to assess the accuracy of a method, a completely independent, reliable and accurate assay being required to establish the absolute quantity of material present in the samples assayed. This is particularly true with biological materials.

Precision is readily estimated by standard statistical techniques, and is often expressed as standard deviation or coefficient of variation, since it is a measure of the spread of results.

Technicon Chart Reader. This was used exactly as described in the Technicon Manual.

Semi-log plots. Standard curves were drawn on semi-log paper, \% $\mathrm{T}$ being plotted on the ordinate which was the logarithmic scale, but inverted. The straight lines were visually fitted to the points so plotted.

Wang technique. Using a Wang 370 programmable electronic calculator (Wang Europe, St. Bryde Street, East Kilbride) optical density was calculated from \% and the best-fit straight line standard curve computed by the least squares method (Snedecor, 1965 ). The concentration of nitrogen in the sample was then computed from the equation of the standard curve.

The only manual steps in this procedure are reading of $\% \mathrm{~T}$ and entry of data into the calculator. Selection of the correct programme is machine checkable. A further refinement of this method was using a D-mac Minmac co-ordinate reader (D-mac, Hillington, Glasgow) to measure $\% \mathrm{~T}$, and also to give a check that no peak has been missed or read twice. Although the Minmac has an interface which allows direct transmission of signals to the Wang calculator system for computation, we have, as yet, used it only for measuring $\% \mathrm{~T}$ with subsequent manual entry to the calculator.

Comparison between the various methods was made by Fisher's F-test of variance ratio (Snedecor, 1965 ), since we are testing for differences in variability and since the true mean of each of the groups compared is the same.

Table I compares the Chart Reader with the semi-log plot method. In this experiment ten colorimetry standards of various nitrogen content were assayed in random order and the nitrogen content found was expressed as a percentage of the stated nitrogen content, i.e. 'per cent recovery of random standards'. Each of the authors made his own comparison. One found a significant difference between the two techniques, the other did not. The maximum $95 \%$ probability range occurred with the Chart Reader, and was almost a $7 \%$ spread on each side of the mean.

A similar comparison of the semi-log plot technique to the Wang technique was made. In this case twenty-six standards were assayed in random order and a joint assessment of results was made (Table 2). The Wang technique proved marginally more precise.

One factor which may influence operator error in manual transcription techniques is the physical size of the abscissa. We have investigated this point both for the 
Table I. Comparison of Chart Reader with semi-log standard curve. Per cent recovery of ten standards assayed in random order (mean $\pm \mathrm{s \Sigma})$. Recoveries were measured independently by two operators, $a$ and $b$

$\begin{array}{ccc} & \text { Recovery } & \text { F } \\ \text { (a) Chart Reader } & 102 \cdot 0 \pm 1 \cdot 1 & 6.25 \\ \text { Semi-log plot } & 99.7 \pm 0.44 & \\ \text { (b) Chart Reader } & 102.5 \pm 0.57 & 1.78 \\ \text { Semi-log plot } & 101.7+0.76 & \\ \text { F }_{\mathbf{0} \cdot 05}=3.18 & & \end{array}$

Table 2. Comparison of semi-log standard curve with statistically fitted optical density standard curve and subsequent calculation of concentration from the curve equation. Per cent recovery of twenty-six standards assayed in random order (mean $\pm \mathrm{SE})$

\begin{tabular}{|c|c|c|}
\hline & Recovery & $F$ \\
\hline Semi-log plot & ror $7 \pm 0.65$ & I. 65 \\
\hline Calculator & $10 \mathrm{I} 50.5 \mathrm{I}$ & \\
\hline
\end{tabular}

Table 3. Effect of scale of abscissa on precision using chart reader. Per cent recovery of thirty standards assayed in random order (mean 上se)

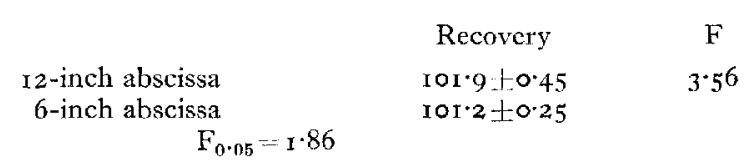

Chart Reader and for the semi-log plot. We used the random standard technique for the Chart Reader, and the results are shown in Table 3 . A marked difference in precision can be seen, there being a smaller spread with the shorter abscissa.

In the semi-log plot assessment, we also investigated the effect of signal size. We prepared two standard curves and randomly selected $\% \mathrm{~T}$ values between 30 and 90 and then asked cach of ten staff members to measure the corresponding concentration from the two graphs. The results are shown in Table 4. Two interesting results are apparent. Firstly, for most of the signals, there are significant differences

'Table 4. Effect of scale of abscissa on precision using semi-log plot. Mean $\pm \mathrm{SE}$ for concentrations corresponding to five different $\% T$ signals as read from pre-drawn graph by ten different subjects

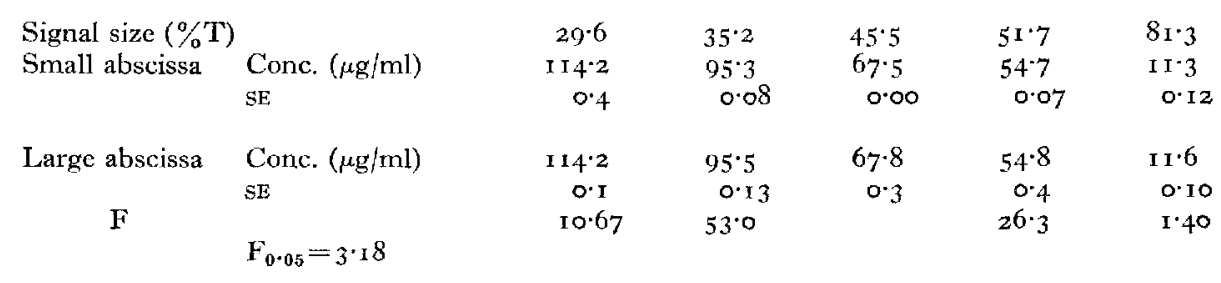


in precision between the two techniques, the smaller scale being more precise except for the $29.6 \% \mathrm{~T}$ signal. Secondly, for one signal $(45.5 \% \mathrm{~T}$ on the small abscissa paper) the corresponding concentration was almost exactly on one of the vertical grid lines and everyone gave the same result. The widest $95 \%$ probability range in this experiment was $\pm 2.5 \%$ but there were three individual results which were between 5 and $10 \%$ away from the mean, almost certainly due to misreading of the graph scale.

In an attempt to make some allowance for accuracy in this study, we selected at random eighteen work sheets covering a period of almost 2 years and, after preparing individual standard curves for each run, calculated the apparent nitrogen content of the standards. Also the apparent $\mathrm{N}$ content of the standards was found using the Wang technique. The results are shown in Table 5. The maximum average deviation of the found $\mathrm{N}$ content from the stated content was $0.9 \%$ for the semi-log plot and $0.8 \%$ for the calculated method. However, there were significant differences in precision for the lowest standard and for the two highest standards. The Wang was slightly the worse for the $10 \mu \mathrm{g} / \mathrm{ml}$ standard, but markedly the better for the 100 and $120 \mu \mathrm{g} / \mathrm{ml}$ standards.

Table 5. Precision of recall of standards from eighteen work sheets, using the semi-log plot technique or the calculator technique

\begin{tabular}{|c|c|c|c|}
\hline \multirow[b]{3}{*}{ Stated conc. $(\mu \mathrm{g} / \mathrm{ml})$} & \multicolumn{3}{|c|}{ Found concentration $(\mu \mathrm{g} / \mathrm{ml})$} \\
\hline & Semi-log plot & Calculator & \\
\hline & Mean $\pm S E$ & Mean SE & $\mathbf{F}$ \\
\hline 10 & $10.1 \div 0.07$ & $9 \cdot 9 \pm 0.11$ & $2 \cdot 30$ \\
\hline 20 & $20.0 \pm 0.07$ & $10.9 \pm 0.08$ & $1 \cdot 00$ \\
\hline 40 & $39 \cdot 8+0.14$ & $39.9+0.09$ & $2 \cdot 20$ \\
\hline 60 & $60.1 \pm 0.15$ & $60 \cdot 2 \pm 0 \cdot 16$ & $\mathbf{I} \cdot 07$ \\
\hline 80 & $79 \cdot 9 \pm 0.14$ & $80 \cdot 2 \pm 0 \cdot 18$ & $1 \cdot 73$ \\
\hline 100 & $99.8 \pm 0.19$ & $100.1 \pm 0.11$ & $2 \cdot 91$ \\
\hline 120 & $119.4 \pm 0.30$ & I $19 \cdot 8+10.13$ & 5.93 \\
\hline
\end{tabular}

To date we have only been able to use the Minmac co-ordinate reader on one occasion. This gave an insignificant improvement in precision compared with manual peak reading (Table 6 ).

Table 6. Effect on precision of using co-ordinate reader of recorder peaks to measure $\% T$. Recovery of seven standards; mean $\pm \mathrm{sE}$. Concentrations were found using the calculator technique

\begin{tabular}{lcc} 
Manual reading & Recovery & $\mathrm{F}$ \\
Co-ordinate reader & $100.5 \pm 0.63$ & $1 \cdot 39$ \\
\multicolumn{1}{c}{$\mathrm{F}_{0.05}=4.28$} & $100.2 \pm 0.53$ &
\end{tabular}

The results presented above clearly show the possible effects on precision of manual transcription techniques. Two operators produced quite different results for precision when comparing two methods of transcription (Table I) and we found 
some gross errors in a simple graph-reading exercise in which the readers were aware that the results were to be analysed for correctness.

On the whole the gain in precision in going from the non-linear, hand-drawn chart technique (where the main error is probably in absolute accuracy) to the semilog computed standard curve is fairly small, but the importance of precision was shown to us in our routine laboratory serum calcium estimations, in which transcription is carried out by the Wang technique. Comparison of recovery of a quality control serum for runs over a period of 3 months in which transcription was by manually drawn graphs with those from 3 months of the Wang technique showed a halving of the coefficient of variation from about $7 \%$ to $3.5 \%$.

W. J. T. gratefully acknowledges financial support from the Medical Research Council.

REFERENCES

Fleck, A. (1967). Proc. Ass. clin. Biochemists 4, 212.

Snedecor, G. W. (1965). Statistical Methods, $5^{\text {th }}$ ed. Iowa: Iowa State University Press.

\title{
Determination of nitrogen in trichloroacetic acid filtrates of milk using
} the Technicon AutoAnalyzer

\author{
By E. J. Hindle and J. V. WheELock, School of Biological Sciences, The University, \\ Bradford, Yorks
}

We have successfully adapted an automated procedure for the estimation of nitrogen in trichloroacetic acid filtrates of milk. These milk filtrates contain numerous small peptides, free amino acids, urea, creatinine and, with low concentrations of trichloroacetic acid, some whey proteins. The $\mathrm{N}$ content varies from $200 \mathrm{mg} / \mathrm{l}$ in a $12 \%(\mathrm{w} / \mathrm{v})$ trichloroacetic acid filtrate to $1000 \mathrm{mg} / \mathrm{l}$ in a $2 \%(\mathrm{w} / \mathrm{v})$ filtrate. As in the Kjeldahl procedure, the organic $\mathrm{N}$ must first be converted into ammonium sulphate. The ammonia released is then estimated colorimetrically. To obtain a satisfactory method for our samples, it was necessary to make a number of modifications in the recommended Technicon procedure for total $\mathrm{N}$.

For digestion, the amount of selenium dioxide-sulphuric acid-perchloric acid mixture and the temperature are critical. Use of two or three acidflex tubes, as recommended, results in a large excess of digestion mixture being introduced so that when the sodium hydroxide is added for neutralization, excess sodium sulphate forms and blocks the tubes. We have found that one Acidflex tube (size $0.08 \mathrm{I}$ in) is sufficient. If the temperature of digestion is too high some of the ammonium sulphate is decomposed and also some catalyst precipitates when water is added to dilute the sample at the end of digestion. If the temperature is reduced too much there is again incomplete digestion. These problems can be overcome by using a 